\begin{tabular}{|c|c|c|}
\hline \multirow{2}{*}{$\begin{array}{r}\text { Case Reports in } \\
\text { Gastroenterology }\end{array}$} & \multicolumn{2}{|c|}{ Case Rep Gastroenterol 2013;7:134-139 } \\
\hline & $\begin{array}{l}\text { DOI: 10.1159/000345961 } \\
\text { Published online: March 13, } 2013\end{array}$ & $\begin{array}{l}\text { (c) } 2013 \text { S. Karger AG, Basel } \\
1662-0631 / 13 / 0071-0134 \$ 38.00 / 0 \\
\text { www.karger.com/crg }\end{array}$ \\
\hline
\end{tabular}

\title{
Secondary Sclerosing Cholangitis due to Gallbladder Adenocarcinoma
}

\author{
David Sicong Fan Serge Alexander Sorser Roberto Mauro Gamarra \\ Providence Hospital \& Medical Center, Southfield, Mich., USA
}

\section{Key Words}

Secondary sclerosing cholangitis · Gallbladder adenocarcinoma - Endoscopic retrograde cholangiopancreatography

\begin{abstract}
Sclerosing cholangitis is a chronic cholestatic liver disease defined by both inflammatory and fibrotic changes of the biliary tract leading to diffuse stricture formation. This entity exists in both a primary and secondary form. Here we present a rare case of secondary sclerosing cholangitis due to direct metastasis from a gallbladder adenocarcinoma. A 55-year-old morbidly obese male presented electively with a 2-week history of low back pain and scleral icterus for 2 days. He also described severe epigastric pain that worsened postprandially and a $13 \mathrm{~kg}$ weight loss over the previous month. The patient denied any personal or familial history of malignancy or prior liver disease. Laboratory evaluation revealed mild elevation of transaminases with moderately elevated alkaline phosphatase and total bilirubin. Imaging included ultrasound and contrast-enhanced computed tomography of the abdomen and pelvis showing multiple large gallstones and a large tissue density mass within the fundus of the gallbladder. Subsequent endoscopic ultrasound was performed revealing celiac and portal lymphadenopathy with fine needle aspirations demonstrating adenocarcinoma. Over the next 15 days, bilirubin progressively increased. Magnetic resonance cholangiopancreatography was unremarkable. Liver biopsy, performed to exclude other etiologies of liver failure, demonstrated biliary cholestasis. Endoscopic retrograde cholangiopancreatography was then performed and an occlusion cholangiogram revealed diffuse multifocal stricturing of the intrahepatic bile ducts and moderate stenosis of the common bile duct without proximal ductal dilatation. Thus secondary sclerosing cholangitis due to gallbladder adenocarcinoma was diagnosed.
\end{abstract}




\section{Introduction}

Secondary sclerosing cholangitis (SSC) is a rare form of sclerosing cholangitis with similar clinical features to that of primary sclerosing cholangitis (PSC). Unlike PSC, there is an identifiable underlying etiology for the development of SSC. The diagnosis of SSC is conducted via three analytical pathways: serologic testing, radiological imaging and histological analysis. While there are an enumerable number of causes, these can be divided into five categories: infectious, toxic, immunological, ischemic and chronic obstructive. The only definitive treatment for most advanced cases is liver transplantation. The prognosis of this disease can vary, but has been shown to have a direct correlation to the timing of diagnosis as well as the underlying etiology. Here we present a rare case of SSC due to gallbladder adenocarcinoma.

\section{Case Report}

A 55-year-old morbidly obese male, with an underlying history of hypertension and dyslipidemia, presented electively, on an outpatient basis, with a 2-week history of low back pain. On presentation, the patient also noted yellowing of the skin for the preceding 2 days and epigastric pain that worsened postprandially, with a $13 \mathrm{~kg}$ weight loss over the previous month. He denied pruritus, fever, chills or changes in bowel habits. There was no personal or familial history of malignancy or liver disease.

One week prior to hospital admission, laboratory evaluation ordered by the patient's primary care provider had revealed: aspartate aminotransferase $58 \mathrm{U} / \mathrm{l}$ (normal 7-40), alanine aminotransferase $85 \mathrm{U} / \mathrm{l}$ (normal 7-40), alkaline phosphatase $168 \mathrm{U} / \mathrm{l}$ (normal 38126), and total bilirubin $1.1 \mathrm{mg} / \mathrm{dl}$ (normal 0.1-1). On admission to hospital, the patient's laboratory studies had increased, with aspartate aminotransferase of $104 \mathrm{U} / \mathrm{l}$, alanine aminotransferase of $153 \mathrm{U} / \mathrm{l}$, alkaline phosphatase of $328 \mathrm{U} / \mathrm{l}$, and total bilirubin of $7.7 \mathrm{mg} / \mathrm{dl}$. Serologic evaluation for chronic liver disease, including autoimmune etiology, was unremarkable. Ultrasonography and computed tomography revealed multiple gallstones and a normal-caliber biliary tree without evidence of ductal dilatation. However, note was made of the presence of a tissue mass in the fundus of the gallbladder with portal and celiac lymphadenopathy (fig. 1). Endoscopic ultrasound with fine needle aspiration confirmed multiple enlarged portal and celiac lymph nodes. The pancreas appeared unremarkable without masses or cystic lesions. The common bile duct was normal in caliber, without evidence of extrinsic compression. Fine needle aspiration of both lymph node groups revealed adenocarcinoma. This finding led to the belief that the fundic mass was a gallbladder adenocarcinoma with nodal involvement.

Over the course of 15 days, liver serologies had increased, with the most drastic rise in total bilirubin to $30 \mathrm{mg} / \mathrm{dl}$. In order to exclude primary or metastatic hepatic pathology, a liver biopsy was performed, revealing benign tissue with characteristics of biliary cholestasis secondary to extrahepatic obstruction. There was no evidence of metastatic involvement of the liver or other hepatic pathologies (fig. 2). Therefore, by serology, invasive and noninvasive imaging as well as histopathology, no other etiology of liver disease was noted. Endoscopic retrograde cholangiopancreatography (ERCP) with an occlusion cholangiogram revealed multiple and severe diffuse strictures of the intrahepatic bile ducts consistent with sclerosing cholangitis (fig. 3). Moderate stenosis of the common bile duct and common hepatic duct was visualized without proximal ductal dilatation. Therefore, without evidence of 
other concurrent pathology, it was believed that the gallbladder lesion was the primary site of malignancy and was the cause of the SSC.

\section{Discussion}

The cholangiographic features described above depict the classic picture of diffuse multifocal biliary strictures with a bead-like appearance of alternating segments of dilated and normal duct, consistent with sclerosing cholangitis. While a hepatic cause for the clinical presentation cannot be excluded, the findings of an otherwise unremarkable liver biopsy make the argument unlikely. In this case, the acuity of clinical symptoms does not fit the typical duration for the pathogenesis of PSC, which has a noted onset of 2 years before the diagnosis is made. This is also supported by the patient's relatively unexceptional medical history, particularly given the lack of any associated autoimmune diseases. Lastly, the presence of gallbladder adenocarcinoma, a logical secondary cause of the cholangitis, further supports the conclusion of SSC as a result of the metastatic gallbladder adenocarcinoma.

Sclerosing cholangitis is a chronic cholestatic liver disease characterized by progressive inflammatory and fibrotic changes of the biliary system resulting in ductal wall thickening and diffuse stricture formation. The development and progression of SSC is the result of repeated cholangiocyte proliferation and apoptosis. This in turn causes and potentiates progressive inflammation and fibrosis. However, unlike PSC where the treatment approach can be varied and unfocused due to the inconsistent understanding of how the disease arises, SSC, by definition, must originate from a known pathological process [1]. With better comprehension and understanding of SSC, a faster and more effective management plan can be formulated; this may also provide better insight into deciphering the pathogenesis of PSC.

While there has been a surge of discussion on the topic, the true prevalence of this disease is either unknown or possibly underestimated. In 2005, a retrospective review by the Mayo Clinic revealed only 31 cases of SSC over a 10-year period [2]. In the medical literature, SSC has been commonly associated with intra-arterial chemotherapy infusion (fluorodeoxyuridine) [3]. However, numerous other causes exist, and these can be broadly divided into five categories: infectious, toxic, immunological, ischemic and chronic obstructive [4].

Infectious causes of sclerosing cholangitis rely on the direct induction of cholangiocyte apoptosis [5]. Implicit in these cases is that the patients are unable to clear the infection due to being in an immunocompromised state. Reported examples include cryptosporidium-, cytomegalovirus- and candida-induced cholangitis after liver transplantation [6, 7]. Toxic causes mediate development of sclerosing cholangitis via direct injury to the biliary epithelium, with the use of formaldehyde for eradication of echinococcal cysts being commonly implicated [8]. Examples of sclerosing cholangitis secondary to immunologically induced inflammation include autoimmune pancreatitis, hypereosinophilic syndrome and mast cellrelated cholangiopathy $[9,10]$. Etiologies that are considered ischemic in nature induce disease via direct compromise of arterial blood flow. These include intra-arterial chemotherapy and operative damage of blood supply during liver transplantation [11]. Lastly, the chronic obstructive etiology mediates stricture formation via chronic biliary obstruction resulting in recurrent cholangitis [4]. The most common causes include choledocholithiasis, biliary stricture secondary to surgical trauma and neoplasia [4].

Recently, a new type of sclerosing cholangitis has garnered some attention. Known as sclerosing cholangitis in critically ill patients (SC-CIP), this entity does not fall accurately under any of the five aforementioned categories as the pathogenesis does not appear to be 
associated with any underlying pathologies [4]. SC-CIP has also been shown to deteriorate much faster and is associated with a higher mortality rate [4].

The initial clinical manifestations of SSC can be quite variable, and most patients are asymptomatic [1]. At this stage, only serologic derangements are noted. However, as the disease progresses and begins to manifest itself, patients typically present with signs and symptoms of chronic cholestasis including jaundice, right upper quadrant abdominal discomfort, pruritus, nausea and vomiting.

In addition to the clinical presentation, further diagnostic steps include serum gammaglutamyl transpeptidase and alkaline phosphatase, ultrasound, magnetic resonance cholangiopancreatography and liver biopsy to exclude underlying liver pathologies, with the gold standard for diagnostic and therapeutic intervention being ERCP. All of the diagnostic steps are necessary to rule out SSC if PSC is suspected. A common finding on ERCP correlating with sclerosing cholangitis is the appearance of multifocal strictures with intermittent segments of dilated biliary ducts on cholangiogram. Another finding more specific to SC-CIP is the evidence of biliary casts [4].

The treatment and management of end-stage hepatic failure secondary to sclerosing cholangitis is limited at best, with the only consensus being orthotopic liver transplantation. Without orthotopic liver transplantation, the overall survival of patients with SSC compared to those with PSC was reduced by up to 17 months [2]. Fortunately, due to the nature of SSC, other therapeutic measures may be undertaken to reverse the secondary causes of sclerosing cholangitis. In one case where sclerosing cholangitis developed as the result of chemotherapy, a novel approach of surgical resection of the biliary stricture was used [12].

Upon review of the literature, cases similar to ours were reported in patients with a history of metastatic disease to the biliary system. One report describes three incidences of sclerosing cholangiopathies in the presence of metastatic adenocarcinoma of the liver [13]. Another case of SSC due to metastatic prostate cancer without evidence of obstructive processes seen on ERCP has also been reported [14]. While it is possible that these cases and the one we present could be considered examples of SSC with an underlying chronic obstructive etiology, we consider the etiology of this cholangiopathy as a direct metastatic infiltration of the biliary tree.

\section{Conclusion}

SSC is a rare but fatal disorder of the biliary tract if not recognized early in the disease process. The diagnosis is one of exclusion, requiring a combination of serologic, histological and radiological features. With early identification, there can be drastic changes in the management as well as a significant improvement in the patient's prognosis [15]. To the best of our knowledge, this is the first case describing SSC as a result of metastatic spread from a primary gallbladder adenocarcinoma.

\section{Disclosure Statement}

The authors have no conflicts of interest to declare. 
Fan et al.: Secondary Sclerosing Cholangitis due to Gallbladder Adenocarcinoma

\section{References}

1 Abdalian R, Heathcote EJ: Sclerosing cholangitis: a focus on secondary cause. Hepatology 2006;44:10631074.

2 Gossard AA, Angulo P, Lindor KD: Secondary sclerosing cholangitis: a comparison to primary sclerosing cholangitis. Am J Gastroenterol 2005;100:1330-1333.

3 Watt K: Primary sclerosing cholangitis and other cholangiopathies; in Yamada T, Alpers D (eds): Textbook of Gastroenterology, ed 5. Hoboken, NJ, Wiley-Blackwell, 2008, pp 1978-2000.

4 Ruemmele P, Hofstaedter F, Gelbmann CM: Secondary sclerosing cholangitis. Nat Rev Gastroenterol Hepatol 2009;6:287-295.

5 Chen XM, Gores GJ, Paya CV, LaRusso NF: Cryptosporidium parvum induces apoptosis in biliary epithelia by a Fas/Fas ligand-dependent mechanism. Am J Physiol 1999;277:G599-G608.

6 Yusuf TE, Baron TH: AIDS cholangiopathy. Curr Treat Options Gastroenterol 2004;7:111-117.

7 Kniepeiss D, Wagner D, Krause R, et al: Mycosis as a cause of secondary sclerosing cholangitis requiring liver retransplantation. Transplantation 2011;91:e14-e16.

8 Sahin M, Eryilmaz R, Bulbuloglu E: The effect of scolicidal agents on liver and biliary tree (experimental study). J Invest Surg 2004;17:323-326.

9 Zhang L, Lewis JT, Abraham SC, et al: IgG4+ plasma cell infiltrates in liver explants with primary sclerosing cholangitis. Am J Surg Pathol 2010;34:88-94.

10 Miura F, Asano T, Amano H, et al: Resected case of eosinophilic cholangiopathy presenting with secondary sclerosing cholangitis. World J Gastroenterol 2009;15:1394-1397.

11 Alazmi WM, McHenry L, Watkins JL, et al: Chemotherapy-induced sclerosing cholangitis: long-term response to endoscopic therapy. J Clin Gastroenterol 2006;40:353-357.

12 Kato Y, Matsubara K, Akiyama Y, et al: Chemotherapy-induced sclerosing cholangitis as a rare indication for resection: report of a case. Surg Today 2009;39:905-908.

13 Vilgrain V, Erlinger S, Belghiti J, et al: Cholangiographic appearance simulating sclerosing cholangitis in metastatic adenocarcinoma of the liver. Gastroenterology 1990;99:850-853.

14 Taylor J, Lindor K: Metastatic prostate cancer simulating sclerosing cholangitis. J Clin Gastroenterol 1993;16:143-145.

15 ter Borg PC, van Buuren HR, Depla AC: Bacterial cholangitis causing secondary sclerosing cholangitis: a case report. BMC Gastroenterol 2002;2:14.

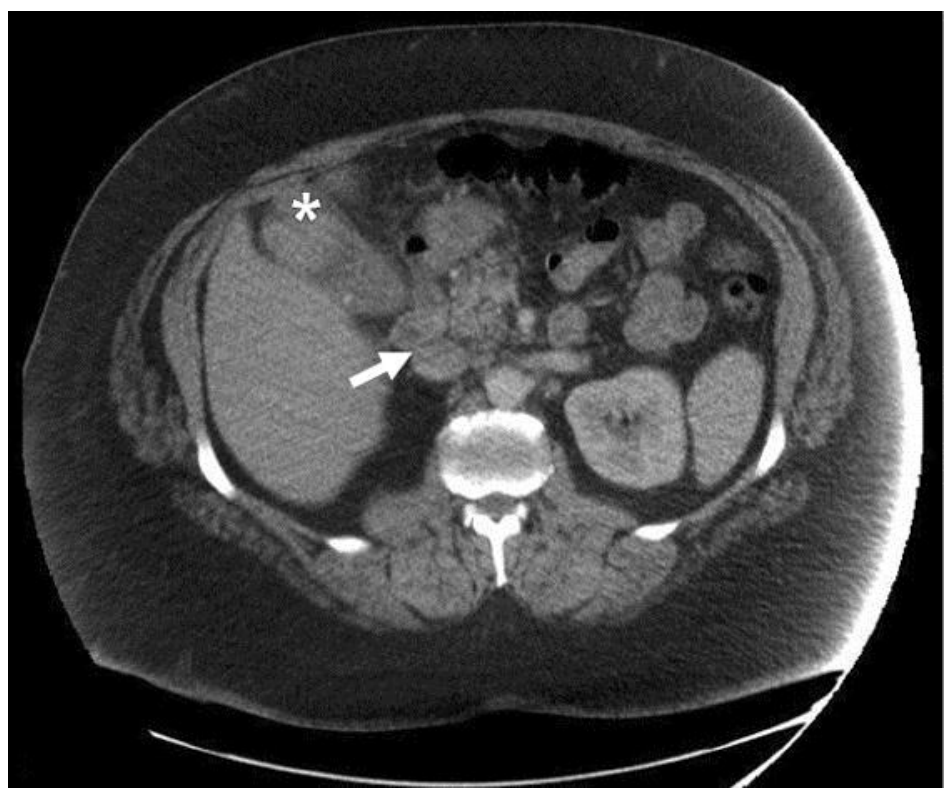

Fig. 1. Cross-sectional image of the abdomen. This section depicts a normal-caliber common bile duct and a large distal tissue mass (asterisk) within the gallbladder. Periportal lymphadenopathy (arrow) is also noted. 


\begin{tabular}{ll|l} 
Case Reports in & \multicolumn{2}{l}{} \\
\cline { 2 - 3 } Gastroenterology & Casep Gastroenterol 2013;7:134-139 & $\begin{array}{l}\text { c 2013 S. Karger AG, Basel } \\
\text { www.karger.com/crg }\end{array}$ \\
\cline { 2 - 3 } & DOI: 10.1159/000345961 & Fan et al. Secondary Sclerosing Cholangitis due to Gallbladder Adenocarcinoma
\end{tabular}

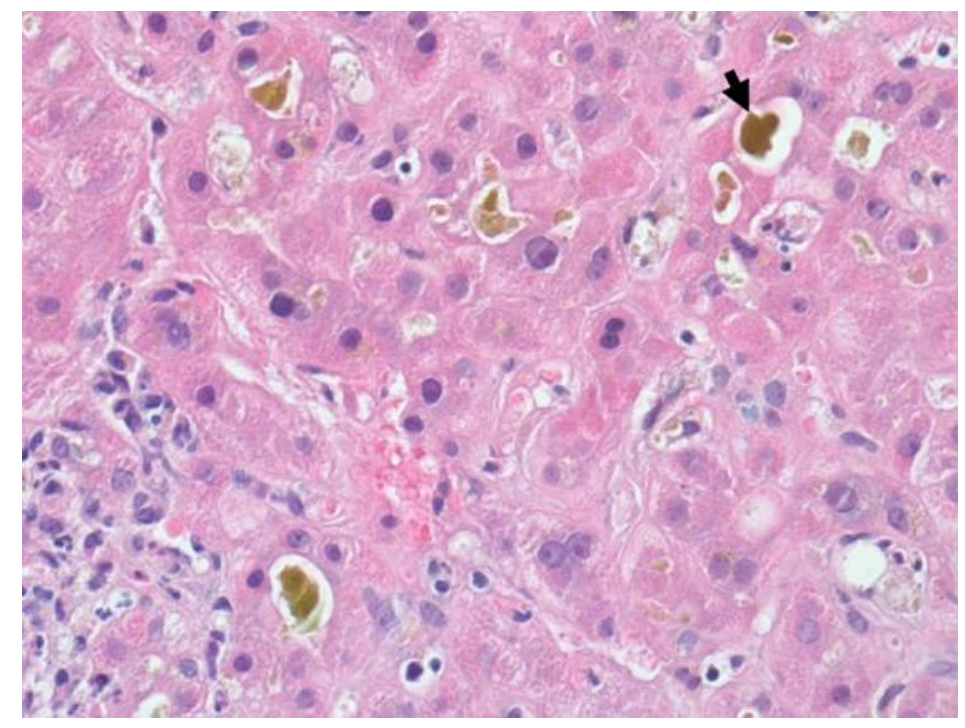

Fig. 2. Histopathological findings from a computed tomography-guided liver biopsy. Histopathological examination with hematoxylin and eosin stain $(\times 100)$ reveals diffuse appearance of fine, foamy hepatocytes (arrow) indicative of biliary cholestasis. There is no evidence of metastatic disease processes involving the liver.

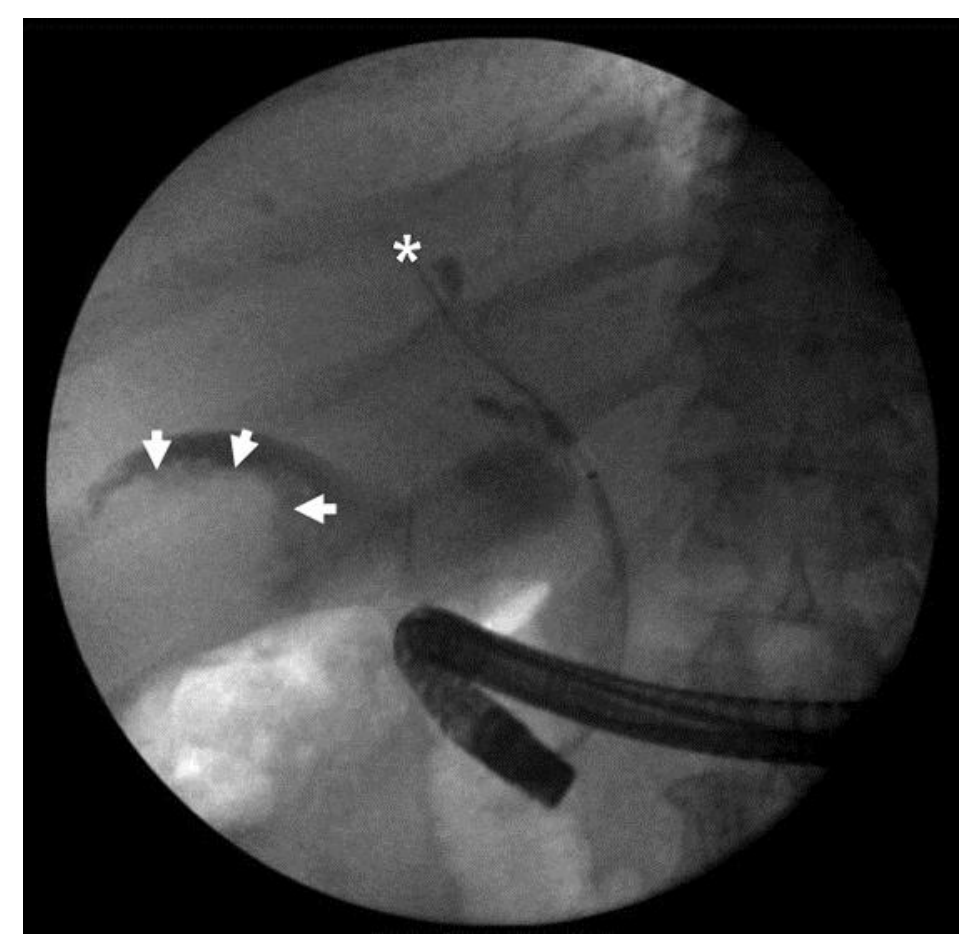

Fig. 3. Occlusion cholangiogram obtained during ERCP showing adequate filling of the cystic duct and evidence of a gallbladder mass (arrows) without visualization of the proximal biliary system (asterisk). 